\title{
The Role of Telemental Health, Tele-consultation, and Tele-supervision in Post-disaster and Low-resource Settings
}

\author{
Eugene F. Augusterfer ${ }^{1} \cdot$ Colleen R. O'Neal $^{2} \cdot$ Shannon W. Martin ${ }^{2} \cdot$ Taiwo Lateef Sheikh $^{3} \cdot$ Richard F. Mollica $^{4}$
}

Accepted: 26 October 2020 / Published online: 28 November 2020

(C) Springer Science+Business Media, LLC, part of Springer Nature 2020

\begin{abstract}
Purpose of Review The goal of this paper was to review recent literature and provide recommendations regarding the use of telemental health, with a focus on tele-consultation and tele-supervision in post-disaster and low-resource settings, including the impact of COVID-19.

Recent Findings The latest research on mental health needs in low-resource settings has identified a high need for mental health services for difficult-to-reach and underserved populations. Research on tele-consultation and tele-supervision was reviewed and found that tele-consultation and tele-supervision to be an effective modality for insuring quality mental health care delivery in low-resource settings. Additionally, two case studies were included which illustrate the use of both tele-consultation and telesupervision in low-resource low- and middle-income settings.

Summary The paper concludes that tele-consultation and tele-supervision hold the promise to narrow the gap in quality mental health services in low-resource settings so often impacted by disaster and conflict. The authors recommend that telemental health training be developed that specifically enhances consultants' and supervisors' skills in tele-consultation and tele-supervision.
\end{abstract}

Keywords Telemental health $\cdot$ COVID-19 $\cdot$ Supervision $\cdot$ Consultation $\cdot$ Disaster response

\section{Introduction}

The sudden onset of the COVID-19 pandemic is a major disaster impacting much of the global population. Health care and mental health providers are being pushed to the limit to meet the demands of their respective populations. Understandably, caring for the medical needs of those suffering from the COVID-19 virus is a priority, but mental health needs are gaining attention in the popular press in addition to

This article is part of the Topical collection on Psychiatry in the Digital Age

Eugene F. Augusterfer

efa@gmhnet.com

1 Harvard Global Mental Health Trauma and Recovery Program, Harvard Medical School, Boston, MA, USA

2 University of Maryland, College Park, MD 20742, USA

3 Ahmadu Bello University, Zaria, Nigeria

4 Harvard Medical School, Boston, MA 02115, USA the medical and mental health press. "The psychological impact of the coronavirus disease 2019 (COVID-19) pandemic must be recognized alongside the physical symptoms for all those affected...Psychological symptoms relating to COVID19 have already been observed on a population level..." [1]. Staffing shortages and lack of specialty training in mental health are a particular challenge in low-resource settings [2]. Thus, mental health consultation and supervision of primary care providers (doctors and nurses) and paraprofessional community health workers is critical. This is especially true in low-resource settings. In low- and middle-income countries, $75 \%$ of people do not get the mental health services they need [3]. (World Health Organization, mhGAP: mental health care in low resource settings) In this paper, we operationalize lowresource settings as low- and middle-income countries as defined by the World Bank, that is, those settings with a disproportionate burden of disease on the poorest individuals in the world's poorest countries [4].

Telemedicine brings the promise of real-time collaboration, consultation, and supervision with health care providers in understaffed, difficult-to-reach, and remote areas. 
Interestingly, with the COVID-19 crisis, the health care provider in need of consultation or supervision might be in the next city block, or thousands of miles away. This paper will focus on the use of telemedicine as a vehicle for consultation and supervision within the mental health specialty area, known as telemental health, telepsychiatry, and, generally, telehealth. Specifically, this paper reviews the use of teleconsultation and tele-supervision as a solution to the problem of providing quality mental health care to those in need, while helping to provide support to on-the-ground mental health care providers. We operationalize tele-consultation as an indirect service that a specialist, such as a psychiatrist, provides as either a one-time or series of consultations, or an opinion, to a primary care provider, who is in need of specialty expertise and problem-solving support on a difficult case [5]. Telesupervision is also an indirect service offered by a mental health specialist, but it involves providing guidance to more junior mental health workers or students in need of supervision and support [6]. For example, in many global lowresource settings, direct care is often provided by community health workers, who may, or may not, have either graduate or post-graduate training, perhaps without much mental health training; thus, a tele-supervisor may provide the worker with the mental health knowledge, skills, and support the worker needs. While we use both terms in this paper, we would like the reader to understand the difference in meaning. The term telemental health (TMH) will be used throughout this paper.

Currently, TMH allows consultants/supervisors to reach distant "hot zones" of the COVID-19 crisis with specialty and expert knowledge that might be lacking in a low-resource setting, or to provide direct TMH services when the frontline medical teams are in need of a reprieve [7]. Thus, TMH is a key component in meeting needs in low-resource settings and extending medical services of frontline providers. This allows expert specialty collaboration with frontline providers who may need expert collaboration and/or supervision. For example, hospitals are using technology and telemedicine to assess patients prior to their arrival at the hospital with what is termed "forward triage," which involves health providers tele-screening patients prior to the patient's arrival at the hospital [7]. Another such innovative screening and diagnostic program was developed at the University of California, Davis Medical Center (UC Davis), which provides tele-consultation to primary care providers (PCPs) in the California agricultural belt [8•]. The program trains PCPs to perform a Structured Clinical Interview for the Diagnostic Statistical Manual (SCID); then, the recorded audio and video is uploaded to a secure server at UC Davis along with the completed SCID results, and reviewed for the purpose of consultation by UC Davis Staff Psychiatrists and Residents. The loop is then completed by providing feedback to the PCPs via tele-consultation regarding diagnosis and treatment recommendations. The entire program is asynchronous which makes consultation more accessible for those with low bandwidth in low-resource settings. This innovative teleconsultation program has proved very successful and can be used as a model for other low-resource settings. In addition to the current COVID-19 crisis, it is important to consider the utility of TMH within the context of other crises. Natural disasters and man-made disasters, including armed conflicts, have marked human existence from time immemorial. However, in recent times, the scale and scope of these events have increased markedly, further solidifying mental health as an issue of global importance. As stated by the World Health Organization (WHO) and the World Bank, the health and mental health consequences of disasters and forced displacement are great [9-11]. Thus, disasters are a major contributor to the more than 264 million people of all ages worldwide estimated to be suffering from depression, making it the leading cause of disability worldwide, and a major contributor to the overall global disease burden [12]. The United Nations reports that disasters, natural and man-made, have caused 70.8 million forcibly displaced persons, including 41.3 million internally displaced persons, 25.9 million refugees, and 3.5 million asylum seekers [13]. These numbers now exceed the number of displaced persons from World War II. Meanwhile, between 1994 and 2013, natural disasters have affected about 218 million people every year [14]. The presence of conflict situations is associated with an increase in mental health disorder prevalence explained by high levels of stress that can prompt psychosocial or psychiatric disorders that were previously nonexistent or dormant [9]. Thus, the rates of mental illness due to man-made and natural disasters have dramatically increased, which is compounded by the fact that the majority of refugees and displaced persons reside in low-resource settings [13].

Most mental health providers working in low-resource settings are without adequate clinical supervision $[15,16]$. Without supervision, mental health support may be of limited quality and exacerbate risks for on-the-ground mental health care providers (e.g., safety concerns, compassion fatigue). For example, Liberia, which has suffered armed conflict, natural disasters (e.g., episodes of extreme flooding), and medical catastrophes, including the Ebola epidemic, has a population of approximately 4.5 million persons, and less than $1 \%$ of the population has access to mental health care [17]. Additionally, Liberia has only two psychiatrists [16]. Thus, most of the mental health care is provided by primary care doctors and mental health care extenders, such as local social workers and community health care workers. Without supervision, such mental health extenders are without support in the field, and thus, their ability to make difficult decisions regarding differential diagnosis and treatments is limited, with consequences for the refugees served. However, as shown by a 7-year longitudinal study with Médecins Sans Frontières (MSF), implementation of TMH supervision has the potential to offer online expertise to the provider in a way that has both clinical value for patient care and educational benefits for providers [18•]. 
As Batada and Leon Solano noted, "One key factor hindering the progress of addressing mental illness lies in the structural imbalance between inadequate capacity to extend support and the high growth rate of individuals requiring treatment... Addressing this gap in a timely and effective manner will warrant innovative approaches that operate in parallel with traditional strategies [19]." Telemental health and consultation/supervision represents one such innovative strategy.

\section{Literature Review}

\section{Mental Health Impact of COVID-19}

The COVID-19 outbreak is leading to additional global health problems such as stress, anxiety, depressive symptoms, insomnia, denial, anger, and fear [20]. As of September 8, 2020, there are over 27.4 million confirmed cases and over 894,000 deaths from COVID-19 worldwide [21]. Communities around the world have implemented social isolation policies in an attempt to slow the spread of the virus, and meanwhile, grief, anxiety, and stress are widespread and increasing [1]. For medical professionals and mental health practitioners, it has become necessary to reach patients in a way that is in line with public health recommendations [22]. While it may take time, resources, and policy changes to integrate telehealth in places where little telehealth infrastructure currently exists, programs and institutions that already have systems in place to support telehealth services are wellequipped to use telehealth as an alternative to in-person treatment, while still allowing for continuity of care [7]. Recent research has shown that for many mental health providers in the USA and Canada, the shift from in-person clinical services to teleservices has been swift and widespread [23].

Additionally, there are numerous reports of health and mental health care providers suffering from exhaustion, fatigue, anxiety, depression, and burnout due to COVID-19 treatment demands (Richard F. Mollica, MD of Massachusetts General Hospital, Harvard Medical School, phone communication, August 2020). Clinical and nonclinical staff are also at risk of psychological distress as they are expected to work longer hours with high risk of exposure to the virus. This may also lead to stress, anxiety, burnout, depressive symptoms, and other problems [1].

\section{Mental Health Impact of Natural and Man-Made Disasters}

Both natural and man-made disasters have impacted the mental health of individuals and communities. For example, Neria and colleagues performed a systematic review of 284 peerreviewed published studies of the mental health impact of natural disasters and concluded that "PTSD among populations exposed to disasters is substantial [24]"; the most consistently documented determinant of the risk of PTSD was the magnitude of exposure to the event. Similarly, a meta-analysis of forty-six published articles examined PTSD rates across 76,101 survivors of earthquakes, and the results estimated that the incidence of PTSD after earthquakes was 23.66\% [25]. Moscona et al. compared medical records at Tulane University Health Sciences Center from 2 years before Hurricane Katrina to 10 years after Katrina to determine the health impact of Katrina [26]. They found significant increases in various medical illnesses, including the following: coronary artery disease (pre $36.4 \%$ vs. post $47.9 \%$ ), diabetes (pre $31.3 \%$ vs. post $39.9 \%$ ), drug abuse (pre $10.2 \%$ vs. post $15.4 \%$ ), and psychiatric illness (pre $6.7 \%$ vs. post $14.9 \%$ ).

Another important area of concern following disasters is the risk of suicide. Alfanso studied suicide rates in Puerto Rico before and after Hurricane Maria in 2017, and he found a $32 \%$ increase in suicides (19 per month pre-Maria vs. 25 per month post-Maria) [27]. Man-made disasters, such as armed conflict, are unique in that the survivors are typically aware that the perpetrators intentionally inflicted the trauma. Survivors of these man-made disasters have often endured unspeakable trauma to themselves and/or their loved ones. As such, the trauma tends to be more complex and, thus, the treatment needs to address the complex nature of the trauma. In a study of Syrian refugees living in a UNHCR refugee camp in Turkey, Jefee-Bahloul et al. surveyed 354 refugees using the "HAD Stress" screening instrument and found that $41.8 \%$ of those surveyed scored consistent with PTSD [28].

\section{The Role of Technology and Telemental Health in Disaster Response in Low-Resource Settings}

Although mental health needs are high in low-resource postdisaster settings [24, 29], high-quality mental health delivery can be challenging to provide; telemental health offers a solution to begin to narrow the gap $[16,30]$. In particular, teleconsultation/supervision in these settings has the potential to strengthen the mental health capacities of local communities and practitioners [31, 32].

In practice, tele-consultation/supervision with lowresource communities has both barriers and advantages [33]. When writing about implementation of TMH in the Middle East, Jefee-Bahloul and Zayour argue that barriers often fall into one of four categories: "cultural, technical, financial, and regulatory" [34]. This appears to ring true across global settings. Barriers often include difficulty with technology (e.g., access to technology and internet; low bandwidth); limited familiarity with or openness to telemental health; scheduling across time zones; and the loss of nonverbal communication 
typically available for in-person interactions [28, 35]. In addition, consultation/supervision often requires cultural competency, with an avoidance of a colonial approach to transcultural consultation/supervision, and an awareness of language differences, cultural expectations and norms, but also attention to differences in ethical codes and regulations $[35,36]$. Indeed, clear expression of emotions and mental health concerns may get lost in transcultural tele-consultation/supervision given cultural differences in emotions, mental health, and feeling self-conscious when speaking to a computer-based video camera. Attention to such cultural and ethical nuances in consultation/supervision may be challenging.

Advantages of TMH in low-resource or post-disaster settings include the opportunity to engage in cross-cultural interactions, flexibility, convenience of meeting time and place, and perhaps most meaningful of all — bringing consultation/ supervision access to people and places that would otherwise have little to no access to mental health supports and interventions [30, 36, 37]. As illustrated in the MSF article described previously [18•], tele-supervision not only supports frontline providers but also serves as an excellent clinical teaching/ training tool. This is especially true in low-resource settings where traditional face-to-face training and educational opportunities may be limited or lacking.

When the bandwidth is inadequate for live consultation/ supervision via synchronous communications, it is possible to have an asynchronous form of consultation/supervision in which the local provider/supervisee uploads a video to a secure server, and the tele-consultant/supervisor uploads a video response. Another form of asynchronous communication could include online forums, where the consultant/supervisor and frontline provider interact via written communication so that each can respond in their own time zone. The asynchronous model also allows each person to read and reflect before responding, to further literature research skills, and to share helpful resources and links via asynchronous communication.

\section{Telemental Health Consultation/Supervision Model}

TMH consultation/supervision can play an important role by providing expert specialty consultation, mentoring, and/or supervision. Serious, and at times life threatening, mental health problems must be addressed by local medical and/or mental health providers who may, or may not, have the training to conduct a proper mental health evaluation and treatment. In keeping with Calderone et al. [38•] "We recommend that telepsychiatry services utilize formal templates and workflow processes that prompt collecting and organizing information required for telepsychiatry consultations...Telepsychiatry consultations offer practical ways to address mental health workforce shortages and provide quality psychiatric care within primary care clinics." The tele-consultation/supervision model below details the mechanism and workflow by which TMH consultation and supervision would ideally work in the field. The following are the steps depicted in the model:

1. A disaster/traumatic event occurs, natural or man-made.

2. The survivor is impacted, both physically and mentally.

3. The impact of the disaster may result in possible mental health problems, including anxiety, depression, PTSD, substance abuse, and/or suicide.

4. The medical and/or mental health provider delivers needed care, including mental health treatment.

5. Tele-consultation/supervision offers mental health expertise and support to the primary care provider, medical and/ or mental health provider.

6. The mental health provider must make sure to have reciprocal communication with the primary medical health provider throughout treatment.

7. The medical and/or mental health provider's treatment, with tele-consultation/supervision support, mitigates the negative impact of the traumatic event on mental health.

The model below depicts the general model of TMH consultation/supervision and has been used in numerous settings with excellent results (see Fig. 1). (Richard F. Mollica, phone communication, August 2020).

\section{Telemental Health Consultation in Low-Resource Countries}

\section{Case Example 1: Psychiatry in Nigeria During the COVID-19 Crisis}

The COVID-19 pandemic has affected low-resource countries in various ways, ranging from lifestyle changes, enforced shutdowns, economic losses, and family dislocations and separations. Low-resource countries are particularly vulnerable, especially for those with existing mental health conditions in addition to everyone affected by stress, anxiety, isolation, loneliness, family pressure, and financial hardship.

Prior to the COVID-19 pandemic, health care in lowresource countries has been burdened by lack of resources, including human, financial, and infrastructure, and this is especially worse for mental health care. Despite the high levels of mental health disabilities, more than $85 \%$ of people with severe mental illness in low- and middle-income countries do not receive the care they require [39-42]. Levels of public expenditure on mental health are very meager in low- and middle-income countries, and more than $80 \%$ of these funds go to mental hospitals [43]; in sub-Saharan Africa, less than $1 \%$ of the health budget is typically spent on mental health [44]. This small allocation is in countries where an already 


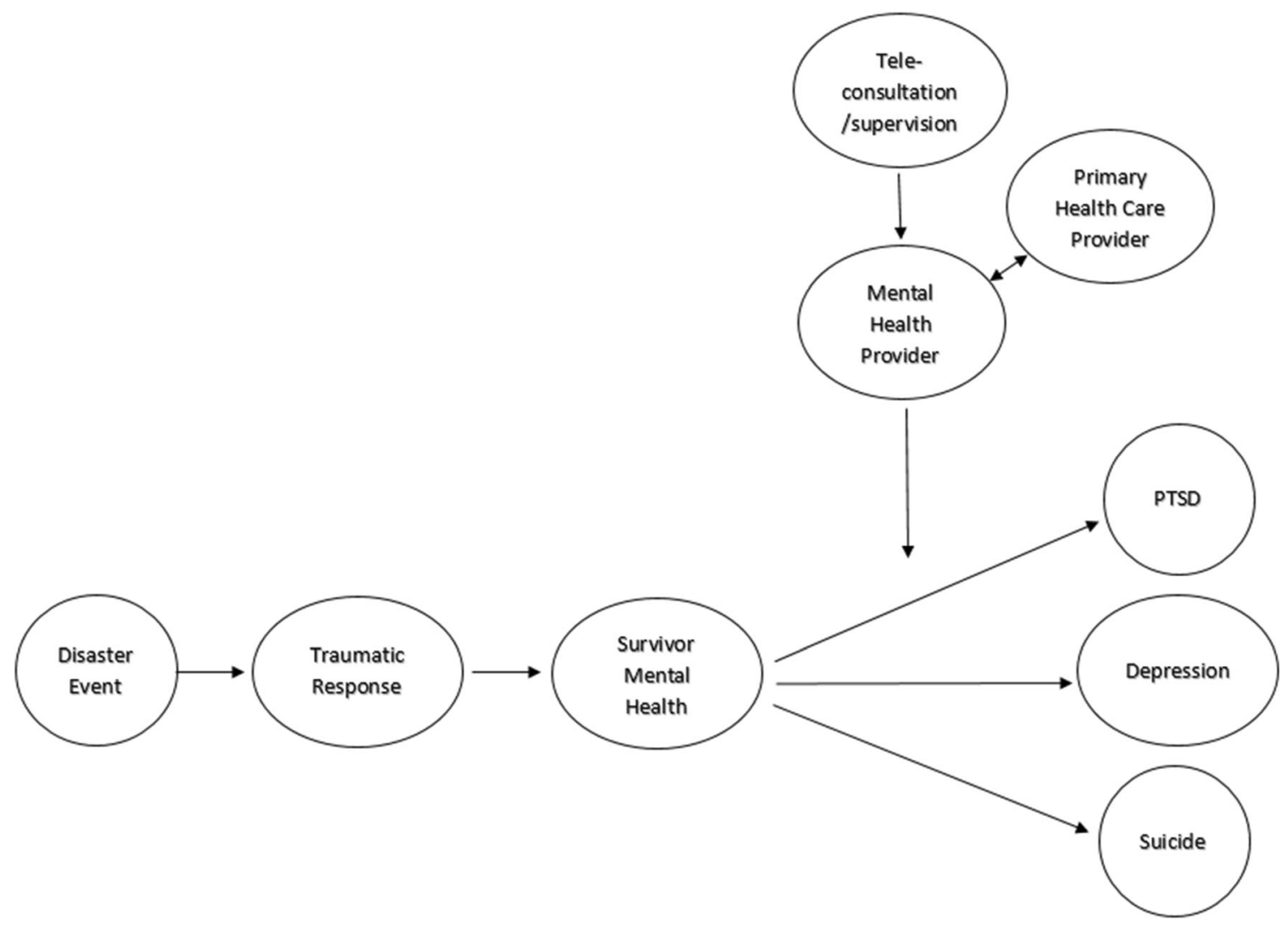

Fig. 1 TMH consultation and supervision model

low proportion of the national budget is spent on health, and is often spent inefficiently, with almost all resources used at tertiary hospital-level services that are inaccessible to the majority of people [45]. Globally, the median number of mental health workers is 9 per 100,000 population, but there is extreme variation (from below 1 in some low-income countries to 72 in high-income countries) [43]. The median number of mental health beds per 100,000 ranges from below 7 in lowand lower-middle-income countries to over 50 in high-income countries. The attendant lockdown and social distancing further exacerbates the existing lack of access to mental health services in the low-resource countries, due to distance of service delivery points, unavailability of transport, fear of contracting COVID-19 at hospitals, and absence of mental health personnel. This may result in situations where individuals who develop mental health problems may not have early intervention while those with existing mental health challenges may experience a worsening of their symptoms as a result of an inability to access services, follow-up consultation, and medication. The need for remote communication technologies, such as the telephone, internet, or videophone, has the potential to mitigate many of these inequalities. The emergence of computer-aided technology alongside growth in the popularity of the internet has increased opportunities for real-time, long-distance consultations [46]. The telephone, in particular, is a widely available telecommunication technology that can be used as a mechanism for support and treatment delivery in low-resource countries. Thus, telemedicine and in particular TMH are helping to level the playing field, especially in resource-poor rural areas.

\section{Telemental Health Consultation in Nigeria During the COVID-19 Pandemic}

In response to this envisaged increase in mental health service demand during the COVID-19 crisis, the Association of Psychiatrists in Nigeria, understanding the dearth of mental health professionals coupled with poor mental health resources, led other mental health professional groups, institutions, non-governmental organizations, civil society organizations, and individuals to form a coalition-Mental Health Professionals for COVID-19 working group. The coalition was tasked with developing a TMH system including psychosocial counseling and mental health consultation help lines. This system provides psychological support and treatment to patients, patient relatives, and frontline health workers in addition to continued mental health support for patients who require follow-up sessions but could not access clinics due 
to lockdowns, patients in isolation wards, and quarantined patients and health workers.

For example, the telemental health system based in the Irrua Specialist Teaching Hospital in Nigeria has an institutional COVID-19 psychosocial response team. The team is led by a Consulting Psychiatrist named Dr. Esther Okogbenin. The team offers TMH treatment and consultations, including mental health and psychosocial support, through dedicated phone lines to patients in isolation wards, relatives in quarantine, and group support sessions to the hospital frontline health workers via zoom conferences. In addition to providing support to patients in remote locations, the Irrua Hospital COVID-19 psychosocial response team provides consultation to frontline health workers, as follows [47]:

Consultation to frontline health workers (primary care provider):

- Details of the primary care provider are given to team experts (tele-consultants) on mental health and psychosocial issues.

- Team expert calls the primary care provider and offers consultation services, such as case consultation related to mental health issues and interventions.

- Team expert gives tips to the primary care provider on self-care and preventive measures as follows:

- Taking care of personal basics, such as proper sleep, diet, and exercise.

- Personal and respiratory hygiene, social/physical distancing, and mask wearing.

- Seek help from appropriate personnel if feeling ill.

This tele-consultation system with frontline health workers embodies "best practices" of TMH interventions in dire situations.

\section{Case Example 2: Post-conflict Refugee Mental Health and Education Using Tele-supervision of Face-to-Face Consultation in Malaysia}

This case example focuses primarily on tele-supervision provided for a face-to-face consultation process. In Malaysia, refugee education is hidden in the shadows because the Malaysian government is not a signatory to the UN convention protecting refugees. Government officials often extort refugees, and the government has a policy of caning and deporting refugees and not allowing refugee children to go to official schools; therefore, refugee children attend informal, under-the-radar refugee schools created by the refugee community [48]. Given refugee students' mental health needs and the lack of training for refugee teachers [49], the Resilient Refugee Intervention program [RRI; 50] has provided, over the past 10 years, indirect emotion and mental health support to refugee students in Malaysia via consultation with the refugee students' teachers, who are largely refugees, themselves. These teachers also face their own mental health challenges, as a result of being refugees and living in a post-conflict country hostile to refugees [50].

The recent RRI iteration in 2019 required intensive weekly tele-supervision due to the equally intensive two-on-one, faceto-face consultation we provided to each of the 105 refugee teacher consultees. The consultations were conducted on-site at their community-based refugee schools hidden, sometimes in plain sight, in apartments, basements, and parking garages all over Kuala Lumpur, Malaysia. As a result of urban traffic, dispersed school sites, and consultation practicum students being scattered across Kuala Lumpur, tele-supervision was necessary even though all of the supervisors lived in Kuala Lumpur. Note that consultation practicum students acted as the consultants, and they were 26 graduate clinical and counseling psychology students whose consultation sessions were audiotaped. Supervisors reviewed their complete audiotapes each week, prior to tele-supervision. In addition, on-line weekly logs were required of each consultation practicum student to prepare them for weekly tele-supervision and facilitate their reflection and fidelity to the RRI manual; however, the fidelity expectations to the RRI manual were loose given the need to be adaptive to refugee teacher needs.

The structure of tele-supervision was based on a structured peer group supervision framework [51] in which the telesupervisor facilitated 30-min case discussions by each of the supervisor's four consultation practicum students. For each case, the first ten minutes were a presentation of demographics, consultation problems, mental health issues, and an audio snippet of the past week's consultation; the second ten minutes involved the presenter being asked clarification questions by the tele-supervisor and peers; and the last ten minutes were suggestions given by the tele-supervisor and peers. Important for the tele-supervision research, we videotaped the tele-supervision sessions via the online platform Skype for future coding. And, for training purposes, supervisors could then be supervised by a lead supervisor via the videotaped supervision sessions.

We strongly believe that RRI could not have been as effective, as our promising initial results have suggested, without tele-supervision to support the provision of quality consultation to prevent mental health problems in refugee teachers and students. Tele-supervision allowed us to reach service providers in disparate locations and permitted fidelity in addition to quality of service delivery by graduate students. The three supervision elements of close weekly tele-supervision, listening to audios uploaded to a secure cloud folder online, and online logs were necessary ingredients for consultation quality. Indeed, a number of the consultation practicum students said that they do not typically receive such supportive, intensive supervision from their other practicum sites, which, 
ironically, provide live, in-person supervision. Perhaps concerns about tele-supervision versus in-person supervision quality are overblown because high-quality supervision seems, to us, to be more dependent on the quality of the supervisor and opportunities for audio review and log reflection, instead of whether or not the supervision is in-person.

It is important, however, that RRI book-ended the telesupervision with, first, an in-person training and, at the end, an in-person final group supervision session and an in-person ceremony. The in-person training and final session were necessary to bond the small supervision groups and ensure the supervision closure process was completed with integrity. The in-person ceremony involved giving US Embassy-printed and embossed RRI completion certificates to each refugee school consultant (tele-supervisees) and consultee.

Although tele-supervision benefits outweighed the challenges, the challenges included the typical Wi-Fi bandwidth problems for certain students. Also, in the beginning of telesupervision, the students seemed initially uncomfortable with the intensive case feedback tele-supervision structure and it is possible that they would have felt more comfortable if the group supervision had been in-person. A future direction for the next iteration of RRI could be to compare the impact of inperson versus tele-supervision.

\section{Discussion and Recommendations}

COVID-19 propelled thousands of mental health providers and consultants/supervisors into isolation from each other. As a result, tele-consultation/supervision has become the new norm of delivery, whether a consultee/supervisee is in a remote or nearby location. The need for quality tele-consultation/supervision support for mental health providers is profound, especially given the mental health consequences for communities impacted by a toxic combination of COVID19, disaster, and/or conflict. Rates of mental health problems, especially PTSD, are much higher after both natural and manmade disasters [27]. For instance, victimization by intentional violence, like in the Syrian conflict, can lead to more complex trauma and high PTSD rates [28]. In low-resource, postdisaster settings, mental health needs are high, availability of in-person consultation/supervision is low, and high-quality mental health delivery is often difficult to provide due to lack of training and educational opportunities [15, 24, 29]. Therefore, tele-consultation/supervision may narrow the gap in quality mental health services in low-resource settings. The novel contribution of this paper is our proposed tele-consultation/supervision model, literature review, and case studies supporting the argument that TMH consultation/supervision can be beneficial for the client, the mental health provider, and the entire mental health system's functioning and effectiveness, especially when the patients and providers are located in hard-to-reach, low-resource settings [18•].

Tele-consultation/supervision is in a strong position to address the mental health service access and quality gap worldwide due to advances in TMH and telecommunications technology. Indeed, tele-consultation/supervision has been found to be beneficial for patient care and provider education [18•]. The TMH consultation/supervision model explained and depicted in this paper is offered as a framework for implementing tele-consultation/supervision in pandemic, disaster, and low-resource settings. The model posits that the provision of tele-supervision to a provider can mitigate the negative impact of disasters and conflict on mental health. An essential mechanism in the model is that the mental health and primary health care providers be in constant communication with each other to avoid miscommunication and provide the best treatment.

Tele-consultation/supervision with low-resource communities, however, has both barriers and advantages [33]. Barriers come from regulatory policies, culture, technical, and financial realms [34]. Also, a concern with tele-supervision, compared to in-person supervision, is that the telesupervisor may have more difficulty being aware of and sensitive to burnout or compassion fatigue [52] in the supervisee. Advantages include cross-cultural interactions, flexibility in scheduling, and improved access to consultation/supervision in low-resource settings.

Quality training in tele-consultation/supervision is necessary. We recommend that tele-health training be further developed around the unique topic of tele-consultation/supervision. Online trainings occur for providing direct services to patients via tele-medicine, but to our knowledge, there are few teleconsultation/supervision trainings. For the development of tele-consultation/supervision training, we suggest a pedagogical structure of community-of-practice groups, with themes for discussion and two leaders. It is important to pay special attention to providing TMH training focused on cultural and ethical competence in tele-consultation/supervision given the potential pitfalls and serious consequences of cross-cultural errors and ethical violations $[35,36]$.

We also recommend consideration of asynchronous consultation/supervision, especially if there are problems with Wi-Fi_ 33 bandwidth in low-resource settings. Quality asynchronous consultation/supervision is ideally conducted with the consultee/supervisee uploading an audio or video of their providing mental health services to the patient. Then, the supervisor would review the supervisee's audio/video and provide feedback to the supervisee. We suggest that the telesupervisor encourages the supervisee's evaluation or progress monitoring of the impact of their intervention on the patient. For instance, there are brief measures, like the Hopkins Symptom Checklist-25 Depression Subscale, that the provider could use on a regular basis to monitor patient progress. 
We have had a serious concern for some time about undertrained and/or young providers providing mental health services in isolated, often dangerous, locations in the field without consultation/supervision support. They deserve such support, and indeed, many have expressed to us that they want tele-consultation/supervision. Many of the providers face significant secondary trauma due to exposure to their patient's traumas resulting from disasters or pandemics; therefore, teleconsultant/supervisor awareness of potential self or supervisee compassion fatigue due to secondary trauma is essential [52].

Another concern is that many providers have not developed a tele-health patient safety protocol. The patient safety protocol in tele-medicine is not the same for in-person versus tele-health sessions. For instance, if a patient becomes agitated or suicidal, then you can keep them safe in a clinic setting, but the same is not true for a tele-health visit. In a tele-health context, a safety protocol would, for example, involve knowing the current location of the patient in order to get them immediate help, if needed. We recommend that the tele-consultant/supervisor encourages and supports their consultee/ supervisee's development of patient safety protocols.

An important lesson from our case studies was that regular tele-consultation/supervision is essential to maintain and improve service quality. A one-time meeting is just not the same as regular, ideally weekly, consultation/supervision, in which not only can quality control be conducted but the supervisee can also build trust and a relationship with the supervisor over time, compared to sporadic or one-time consultation. Such trust is necessary for an open, honest, high-quality consultation/ supervision to be conducted [53]. Also, the Nigerian case example demonstrates the importance of not only the teleconsultant but also of the support provided by the entire teleconsultation system that Nigeria put in place as a coalition to respond to serious mental health demands due to COVID-19.

\section{Conclusion}

In conclusion, the field of TMH needs to increase opportunities for and training in tele-consultation and tele-supervision. The need for tele-consultation/supervision is especially important to increase access to support for mental health providers in low-resource settings that are coping with the impact of disasters, conflict, and COVID-19. Such tele-consultation/supervision provision and training holds implications for access to quality mental health support worldwide, especially during our global pandemic, in low-resource settings, and given the current mass migration crisis.

\section{Compliance with Ethical Standards}

Conflict of Interest Eugene F. Augusterfer, Colleen R. O'Neal, Shannon W. Martin, Taiwo Lateef Sheikh, and Richard F. Mollica declare that they have no conflict of interest.
Human and Animal Rights This article does not contain any studies with human or animal subjects performed by the any of the authors. All authors work is in full compliance with the Helsinki declaration and its amendments, institutional/national research committee standards, and international/national/institutional guidelines.

\section{References}

Papers of particular interest, published recently, have been highlighted as:

- Of importance

1. Zhou X, Snoswell CL, Harding LE, Bambling M, Edirippulige S, Bai $X$, et al. The role of telehealth in reducing the mental health burden from COVID-19. Telemed J E Health. 2020;26:377-9.

2. Tiberi O. Mental health in Haiti: beyond disaster relief, $2016 \mathrm{~J}$ Global Health 6(2):14-21.

3. World Health Organization: WHO Mental Health Gap Action Programme (mhGAP). https://www.who.int/mental_health/ mhgap/en/. Accessed September 21, 2020.

4. Fantom, NJ, Serajuddin, U. The World Bank's classification of countries by income (English). Policy Research working paper; no. WPS 7528 Washington, DC: World Bank Group. 2016. http://documents.worldbank.org/curated/en/ 408581467988942234/The-World-Banks-classification-ofcountries-by-income. Accessed August 2020.

5. Dougherty AM. Psychological consultation and collaboration in school and community settings. 5th ed. Brooks/Cole Cengage Learning: Australia; 2009.

6. Simon DJ, Cruise TK, Huber BJ, Swerdlik ME, Newman DS. Supervision in school psychology: the developmental/ecological/ problem-solving model. Psychol Sch. 2014;51(6):636-46. https:// doi.org/10.1002/pits.21772.

7. Hollander JE, Carr BG. Virtually perfect? Telemedicine for Covid19. N Engl J of Med 2020;382:1679-81. https://doi.org/10.1056/ NEJMp2003539.

8. Yellowlees P, Burke Parish M, González Á, et al. Asynchronous telepsychiatry: A component of stepped Integrated Care. Telemed J E Health. 2018;24(5):375-8. https://doi.org/10.1089/tmj.2017. 0103. A team at UC Davis is conducting a 5-year study to explore the outcomes of an asynchronous model of telepsychiatry consultation. This article presents the baseline characteristics of participants, discusses how the model works, and presents three case study examples demonstrating the effectiveness and efficiency of this approach in practice.

9. World Bank: Psychosocial support in fragile and conflict-affected settings. https://www.worldbank.org/en/topic/ fragilityconflictviolence/brief/psychosocial-support-in-fragile-andconflict-affected-settings. Updated May 9, 2016. Accessed April 8, 2020.

10. World Bank: 2017. Forcibly displaced: toward a development approach supporting refugees, the internally displaced, and their hosts. Washington, DC: World Bank. World Bank. https:// openknowledge.worldbank.org/handle/10986/25016.

11. World Health Organization. Health in the context of migration and forced displacement: Side event report. 2016. https://www.who.int/ docs/default-source/documents/publications/health-in-the-contextof-migration-and-forced-displacement.pdf?sfvrsn=71bbc07d_1. Accessed April 8, 2020.

12. World Health Organization. Depression fact sheet. 2020. https:// www.who.int/news-room/fact-sheets/detail/depression\#: :text= Key facts,overall global burden of disease. Accessed April 3, 2020. 
13. United Nations High Commissioner on Refugees, Global trends in forced displacement. 2019.

14. Wallemacq, P, Guha-Sapir, D, McClean D, CRED, UNISDR. The human cost of natural disasters - A global perspective. 2015.

15. Jefee-Bahloul $\mathrm{H}$. Introduction to telemental health and its use in low-resource settings. In: Jefee-Bahloul H, Barkil-Oteo A, Augusterfer EF, editors. Telemental health in resource-limited global settings. New York: Oxford University Press; 2017.

16. Katz CL, Washington FB, Sacco M, et al. A resident-based telepsychiatry supervision pilot program in Liberia. Psychiatr Serv. 2018;70(3):243-6.

17. World Health Organization. Culture and mental health in Liberia: a primer. Geneva: World Health Organization; 2017.

18. Delaigue S, Bonnardot L, Steichen O, et al. Seven years of telemedicine in Médecins Sans Frontières demonstrate that offering direct specialist expertise in the frontline brings clinical and educational value. J Glob Health. 2018;8(2):020414. https://doi.org/10.7189/ jogh.08.020414. MSF has implemented a telemedicine program with outstanding results in low-resource settings. From 2010 to 2017, a total of 5646 telemedicine cases were included in the study, and they found increased rates of referral, decreased time allocation to each case, and a significant reduction in the time to respond to a case (from $20 \mathrm{~h}$ to $5 \mathrm{~h}$ ). The use of telemedicine for consultation/supervision with specialists who were not in the immediate area was found to have both clinical benefits for patients and educational benefits for practitioners.

19. Batada A, Leon Solano R. Harnessing technology to address the global mental health crisis. Washington, DC: World Bank; 2019.

20. Torales J, O'Higgins M, Castaldelli-Maia JM, Ventriglio A. The outbreak of COVID-19 coronavirus and its impact on global mental health. Int J Soc Psychiatry. 2020;66(4):317-20. https://doi.org/10. 1177/0020764020915212.

21. Coronavirus Resource Center: Johns Hopkins University and Medicine. Updated daily. Accessed September 2020.

22. Whaibeh E, Mahmoud $\mathrm{H}, \mathrm{Naal} \mathrm{H}$. Telemental health in the context of a pandemic: the COVID-19 experience. Curr Treat Options Psychiatry. 2020;7:1-5. https://doi.org/10.1007/s40501-02000210-2.

23. Pierce BS, Perrin PB, Tyler CM, McKee GB, Watson JD. The COVID-19 telepsychology revolution: a national study of pandemic-based changes in U.S. mental health care delivery [published online ahead of print, 2020 Aug 20]. Am Psychol. 2020. https://doi.org/10.1037/amp0000722.

24. Neria Y, Nandi A, Galea S. Post-traumatic stress disorder following disasters: a systematic review. Psychol Med. 2008 Apr;38(4):46780 .

25. Dai W, Chen L, Tan H, Wang J, Lai Z, Kaminga AC, et al. Association between social support and recovery from posttraumatic stress disorder after flood: a 13-14-year follow-up study in Hunan, China. BMC Public Health. 2016;16:194. https://doi.org/ 10.1186/s12889-016-2871-x.

26. Moscona JC, Peters MN, Maini R, Katigbak P, Deere B, Gonzales $\mathrm{H}$, et al. The incidence, risk factors, and chronobiology of acute myocardial infarction ten years after Hurricane Katrina. Disaster Med Public Health Prep. 2019;13(2):217-22. https://doi.org/10. 1017/dmp.2018.22.

27. Alfanso CA. PTSD and suicide after natural disasters. Psychiatr Times. 2018

28. Jefee-Bahloul H, Moustafa MK, Shebl FM, Barkil-Oteo A. Pilot Assessment and Survey of Syrian refugees' Psychological Stress and Openness to Referral for Telepsychiatry (PASSPORT Study). Telemed J E Health. 2014;20(10):977-9. https://doi.org/10.1089/ tmj.2013.0373.

29. Goldmann E, Galea S. Mental health consequences of disasters. Annu Rev Public Health. 2014;35:169-83. https://doi.org/10. 1146/annurev-publhealth-032013-182435.
30. Augusterfer EF, Mollica RF, Lavelle J. Leveraging technology in post-disaster settings: the role of digital health/telemental health. Curr Psychiatry Rep. 2018;20(10):1-8. https://doi.org/10.1007/ s11920-018-0953-4.

31. Johnson DS. Thinking outside the box: are we ready for teleprecepting to expand training and practice venues? Issues Ment Health Nurs. 2019;40(3):215-22. https://doi.org/10.1080/ 01612840.2018 .1488903$.

32. Mehrotra K, Chand P, Bandawar M, Rao Sagi M, Kaur S, G A, et al. Effectiveness of NIMHANS echo blended tele-mentoring model on integrated mental health and addiction for counsellors in rural and underserved districts of Chhattisgarh, India. Asian J Psychiatr. 2018;36:123-7. https://doi.org/10.1016/j.ajp.2018.07. 010

33. Martin P, Kumar S, Lizarondo L. Effective use of technology in clinical supervision. Internet Interv. 2017;8:35-9. https://doi.org/ 10.1016/j.invent.2017.03.001.

34. Jefee-Bahloul H, Zayour Z. Telemental health in the Middle East In: Jefee-Bahloul H, Barkil-Oteo A, Augusterfer EF, editors. Telemental health in resource-limited global settings. New York: Oxford University Press; 2017.

35. Inman AG, Soheilian SS, Luu LP. Telesupervision: building bridges in a digital era. J Clin Psychol. 2019;75(2):292-301. https://doi. org/10.1002/jclp.22722.

36. Duan C, Falender C, Goodyear R, Qian M, Jia X, Jiang G. Telesupervision-of-supervision across national boundaries: United States and China. J Clin Psychol. 2019;75(2):302-12. https://doi. org/10.1002/jclp.22723.

37. Furlan AD, Zhao J, Voth J, Hassan S, Dubin R, Stinson JN, et al. Evaluation of an innovative tele-education intervention in chronic pain management for primary care clinicians practicing in underserved areas. J Telemed Telecare. 2019;25(8):484-92. https://doi. org/10.1177/1357633X18782090.

38. Calderone J, Lopez A, Schwenk S, Yager J, Shore JH. Telepsychiatry and integrated primary care: setting expectations and creating an effective process for success. Mhealth. 2020;6:29. Published 2020 Jul 5. https://doi.org/10.21037/mhealth.2020.02. 01 . This article offers timely insights on effectively using TMH to support psychiatric consultation with primary care providers, from the lens of an integrated psychiatrist. In addition to considering the clinical challenges for telesupervision that arise when substituting face-to-face interactions between professionals with virtual meetings, the article also discusses recommendations for setting expectations, reviewing data, and communicating efficiently.

39. Whiteford HA, Degenhardt L, Rehm J, Baxter AJ, Ferrari AJ, Erskine HE, et al. Global burden of disease attributable to mental and substance use disorders: findings from the Global Burden of Disease Study. 2010. Lancet. 2013;382(9904):1575-86.

40. Vigo D, Thornicroft G, Atun R. Estimating the true global burden of mental illness. Lancet Psychiatry. 2016;3(2):171-8.

41. Demyttenaere K, Bruffaerts R, Posada-Villa J, Gasquet I, Kovess $\mathrm{V}$, Lepine JP, et al. Prevalence, severity, and unmet need for treatment of mental disorders in the World Health Organization World Mental Health Surveys. JAMA. 2004;291(21):2581-90.

42. UN Policy brief: COVID-19 and the Need for Action on Mental Health. 2020. (p2).

43. Mental Health Atlas. 2017. Geneva: World Health Organization; 2018. Licence: CC BY-NC-SA 3.0 IGO.

44. Mental Health Atlas 2014. Geneva: World Health Organization; 2014. p. 2015

45. Saxena S, Thornicroft G, Knapp M, Whiteford H. Resources for mental health: scarcity, inequity, and inefficiency. Lancet. 2007:370(9590):878-89. 
46. Augenstein J. Opportunities to Expand Telehealth Use Amid The Coronavirus Pandemic. Health Affairs Blog, March 16, 2020. https://doi.org/10.1377/hblog20200315.319008.

47. IASC Guidance on Basic Psychosocial Skills-A Guide for COVID19 Responders, Inter-Agency Standing Committee, 26 May 2020.

48. Nathan SS. Because I am a refugee: the denial of human rights to refugee women and girls in Malaysia. In: Thas AK, Menon L, Binti Abdullah MC, Nathan SS, editors. Equality under construction: Malaysian women's human rights report. Selangor: Persatuan Kesedaran Komuniti Selangor; 2013.

49. O'Neal C, Atapattu R, Jegathesan A, Clement J, Ong E, Ganesan A. Classroom management and socioemotional functioning of Burmese refugee students in Malaysia. J Educ Psychol Consult. 2018;28(1):6-42. https://doi.org/10.1080/10474412.2016. 1193740 .

50. O'Neal CR, Gosnell NM, Ng WS, Ong E. Refugee-teacher-trainrefugee-teacher intervention research in Malaysia: promoting classroom management and teacher self-care. J Educ Psychol Consult.
2018;28(1):43-69. https://doi.org/10.1080/10474412.2017. 1287576 .

51. Newman D, Nebbergall A, Salmon D. Structured peer group supervision for novice consultants: procedures, pitfalls, and potential. J Educ Psychol Consult. 2013;23(3):200-16. https://doi.org/10. 1080/10474412.2013.814305.

52. Figley CR. Compassion fatigue: coping with secondary traumatic stress disorder in those who treat the traumatized. New York: Brunner/Mazel; 1995.

53. Newman D. Supervision of school-based consultation training: addressing the concerns of novice consultants. In: Rosenfield SA, editor. Becoming a school consultant: lessons learned. New York: Routledge/Taylor \& Francis Group; 2012.

Publisher's Note Springer Nature remains neutral with regard to jurisdictional claims in published maps and institutional affiliations. 